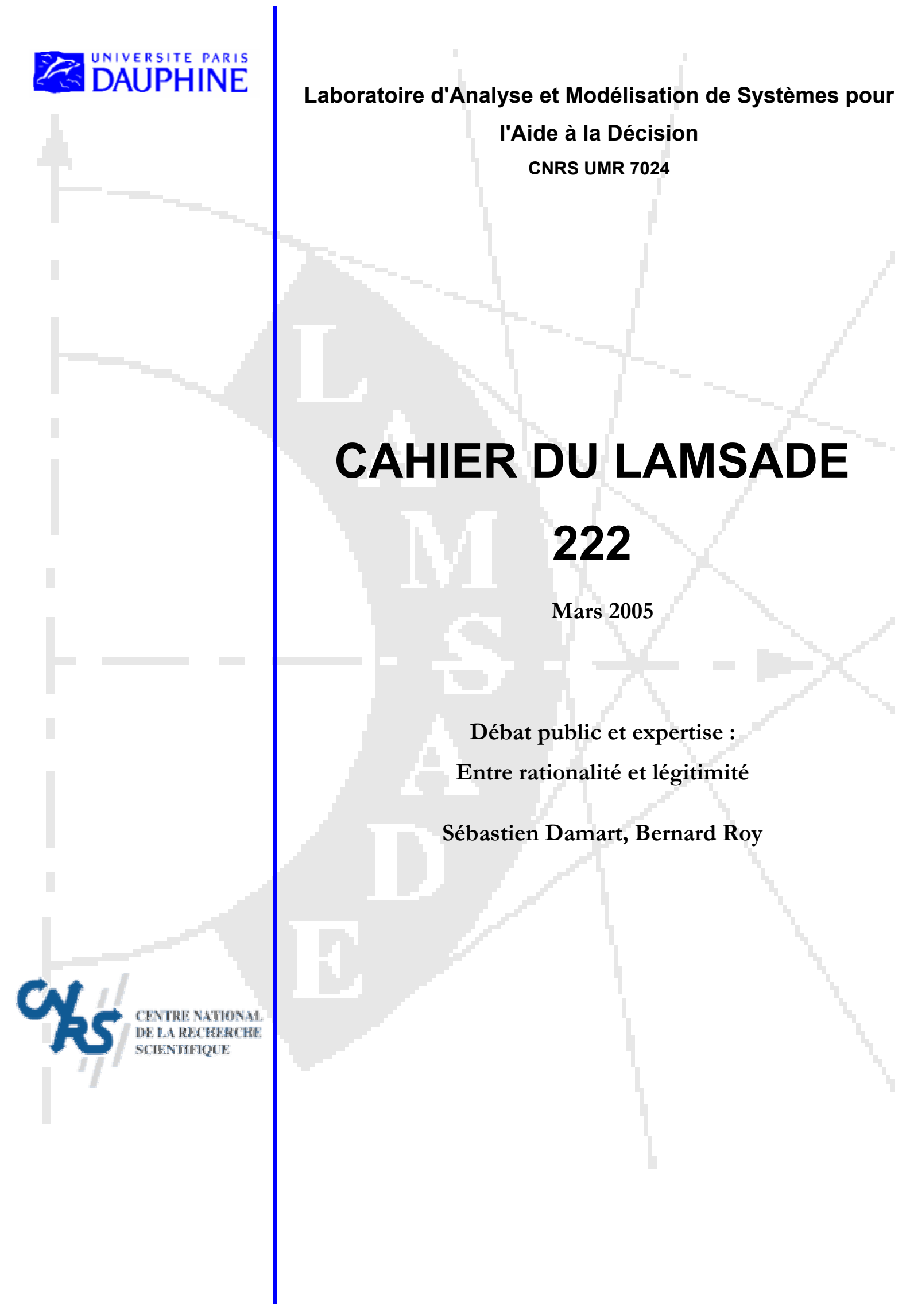




\section{TABLE DES MATIÈRES}

Résumé 2

Abstract 3

1. Introduction 4

2. Définition et principes de l'analyse coûts avantages 6

2.1 Qu'est-ce que l'analyse coûts avantages 6

2.2 Comment l'ACA est-elle réalisée?

2.2.1 La procédure de monétarisation des effets non marchands et effets externes 8

2.2.2 Utilisation des valeurs monétaires, calculs de rentabilité des projets et $\begin{array}{ll}\text { situation de référence } & 10\end{array}$

3. Législation, outils et pratique du débat public 11

3.1 Ce que la loi dit à propos du débat public 11

3.2 Institutions et outils du débat public à l'heure actuelle en France 13

$\begin{array}{ll}\text { 3.2.1 La procédure d'enquête publique } & 13\end{array}$

3.2.2 La Commission Nationale du Débat Public 13

3.3 Une expérience de débat public : le troisième aéroport parisien 14

4. Pratique et valeur des démarches d'ACA, contributions au débat public 15

4.1 Limites de l'ACA au service de la rationalisation des choix publics 16

$\begin{array}{ll}\text { 4.1.1 Objectivité des démarches d'ACA } & 18\end{array}$

4.1.2 Scientificité des démarches d'ACA 17

$\begin{array}{ll}\text { 4.1.3 Biais dans la pratique de l'ACA } & 17\end{array}$

4.2 Calcul économique et débat public 19

5. A la recherche de nouveaux liens entre rationalité et légitimité 21

5.1 Aménagement du processus et renouvellement des outils 22

$\begin{array}{ll}5.1 .1 \text { Mise en place d'une table de concertation } & 22\end{array}$

5.1.2 Instrumentation des premières étapes de la concertation 23

5.1.3 Renouvellement des outils d'évaluation en phase amont 24

5.2 Renouvellement des modes d'utilisation des outils d'expertise actuels 26

$\begin{array}{ll}\text { 5.2.1 Pratique de l'ACA en phase amont } & 26\end{array}$

5.2.2 Enrichir les techniques d'ACA en phase aval 27

$\begin{array}{ll}\text { 6. Conclusion } & 28\end{array}$

Références 29 


\title{
Débat public et expertise : entre rationalité et légitimité
}

\begin{abstract}
Résumé
La question du choix des investissements en infrastructures de transport s'inscrit en fait dans une problématique à deux niveaux. D'un côté, le décideur public dispose de ressources limitées et doit les utiliser de la façon la plus profitable qui soit. Il importe aussi au décideur de savoir faire entrer, dans une évaluation dite "rationalisante " des projets d'investissements, les effets multiples du projet et même ceux difficiles à évaluer. D'un autre côté, au delà de la simple question du choix entre projets d'investissement, c'est celle de l'arbitrage entre différentes catégories d'enjeux qui est discutée. L'autre contrainte du décideur public est ainsi de faire accepter par la collectivité l'ordre de priorité des enjeux tacitement contenus dans les choix publics qu'il fait. Le décideur est ainsi face à deux catégories de préoccupations : rationaliser l'utilisation des ressources publiques et parvenir à des choix les plus acceptables possibles du point de vue de la collectivité.

Le recours important aux outils de calcul économique de type analyse coûts avantages (ACA) s'inscrit dans la réponse du décideur public à la première catégorie d'enjeux. Quant à la seconde, le législateur a tenté depuis plusieurs décennies d'institutionnaliser le débat entre le décideur public et les acteurs parties prenantes.

L'objet de cet article est l'examen de la façon dont s'articulent aujourd'hui en France les pratiques du calcul économique et du débat public. Nous serons amenés à définir et présenter les principes de l'analyse coûts avantages. Nous serons également conduits à montrer brièvement comment le débat public s'est institutionnalisé en France depuis plusieurs décennies. Nous tenterons ensuite de mettre en perspective les difficultés liées à l'usage de l'ACA dans le cadre du débat public : celles-ci concernent, à notre avis, les exigences de rationalité et de légitimité au sein du processus de décision liés aux infrastructures de transport. Nous terminerons enfin en faisant quelques propositions susceptibles de faire évoluer ces pratiques afin que le déroulement du processus parvienne à mieux concilier la recherche conjointe des objectifs de rationalisation des choix d'investissement publics de transport et de légitimation des décisions correspondantes.
\end{abstract}

Mots clés : Analyse coûts avantages, analyse multicritère, débat public, rationalité, légitimité. 


\title{
Public debate and technical expertise: between rationality and legitimacy
}

\begin{abstract}
The questioning of how public investments decisions are made leads to a two-level problematic. On the one hand, public decision makers have constrained resources that they have to use the best way they can. On the other hand, by choosing between alternative investments projects, decision makers are revealing priority choices between different stakes. So, decision makers have to face with two kinds of issues: spending with special care constrained resources and making the most acceptable decisions they can.

In France, for several years, cost benefit analysis has been used to take into account the first issue. The second issue has lead the legislator to rule public expression and stakeholders involvement. This has been a long process made of more or less successful trials.

The purpose of this article is to examine how the use of cost benefit analysis interacts with the practice of public debate and stakeholders' participation. In Section 2 (after an introductory section), we will define more precisely principles of cost benefit analysis. In Section 3, we will see how stakeholders' participation has been progressively ruled in France. Then we will study the difficulties to conciliate cost benefit analysis and stakeholders' participation. At last, we will give some suggestions for conciliating legitimacy and rationality in public investments decisions making process.
\end{abstract}

Keywords: Cost benefit analysis, multicriteria analysis, stakeholder participation, rationality, legitimacy. 


\title{
Débat public et expertise : entre rationalité et légitimité
}

\author{
Sébastien Damart ${ }^{1}-$ Bernard Roy $^{1}$
}

\section{Introduction}

Les réseaux de transports occupent dans la plupart des pays une place de plus en plus importante. Dans le fonctionnement des économies développées, ils jouent un rôle décisif car les flux de marchandises et d'hommes sont de plus en plus intenses. Les composantes des réseaux de transport d'un pays sont nombreuses. Les routes et autoroutes, les lignes de chemin de fer, les passages fluviaux, les gares, les aéroports, etc. constituent les infrastructures d'un maillage complexe qui dessert toutes les parties d'un territoire souvent vaste. Ces composantes sont souvent complémentaires et leur mise en cohérence est un atout majeur pour une puissance industrielle et économique.

Dans les contextes où l'allocation des deniers publics est fortement contrainte se pose naturellement continuellement la double question de la pertinence de la réalisation des projets d'infrastructure et du choix entre les différentes variantes potentiellement réalisables. La question est difficile si l'on choisit de prendre en compte, comme tente de le faire le décideur public, toutes les implications liées à la réalisation ou non d'une infrastructure de transport public. Il s'agit en effet, dans cette perspective, de considérer les coûts financiers d'investissement et d'entretien des infrastructures susceptibles d'être réalisés mais aussi leurs impacts potentiels (positifs et négatifs) sur la collectivité : temps de transport, sécurité, cadre de vie,... sans négliger aucune des modifications possibles de l'environnement que leur réalisation pourrait faire subir aux générations futures. La façon de prendre en compte ces effets sur l'environnement éclaire tout particulièrement la difficulté de l'exercice. Ceux-ci sont multiples et de nature très diverse : impact sur l'esthétique d'un paysage, effets sur l'air (pollution de l'air) ou sur l'environnement sonore (le bruit), conséquences sur les conditions de vie des riverains, etc. La prise en compte de ces effets implique de suivre des démarches complexes et de mobiliser des outils d'évaluation sophistiqués car un bien, un confort ou un désagrément environnemental est difficilement évaluable (cf. encadré $\mathrm{n}^{\circ} 1$ ).

\begin{abstract}
La prise en compte des effets d'une infrastructure de transport sur l'environnement est difficile. Cela est notamment le cas des effets en termes de pollution sonore. Michel Mattheu (Commissariat général au plan) indique ainsi que « (...) la gêne occasionnée par le bruit augmente diverses morbidités mais elle est surtout ressentie comme une pression psychologique et un mal être diffus. Faute de disposer d'un indicateur d'impact incontestable, les experts s'efforcent de pondérer des indicateurs de pression - en fait des niveaux sonores - de façon à approcher au mieux le dommage ressenti par les personnes exposées. A l'occasion des extensions d'aéroports, la pertinence de ces indicateurs est régulièrement remise en cause car certaines enquêtes montrent que la gêne subjective des riverains est mal corrélée avec les indices adoptés. » (Responsabilité et Environnement, Annales des Mines, octobre 2002).
\end{abstract}

En fait, la difficile appréhension de la pollution sonore soulevée dans cet encadré nous montre que le problème du choix des investissements publics est autant affaire de rationalité que de légitimité. La prise en compte d'effets multiples dans le cadre d'arbitrages budgétaires par

\footnotetext{
${ }^{1}$ LAMSADE, Université Paris-Dauphine, Place du Maréchal De Lattre de Tassigny, 75775 Paris cedex 16.
} 
définition contestables inscrit effectivement la question du choix des investissements en infrastructures de transport dans une problématique à deux niveaux. D'un côté, le décideur public dispose de ressources limitées et doit les utiliser de la façon la plus profitable qui soit. Il importe ainsi au décideur de savoir faire entrer, dans une évaluation dite " rationalisante » des projets d'investissements, les effets multiples du projet et mêmes ceux difficiles à évaluer. D'un autre côté, au delà de la simple question du choix entre projets d'investissements, c'est celle de l'arbitrage entre différentes catégories d'enjeux qui est discutée. L'autre contrainte du décideur public est ainsi de faire accepter par la collectivité l'ordre de priorité des enjeux. En résumé, le décideur est face à deux catégories de préoccupations : rationaliser l'utilisation des ressources publiques et parvenir à des choix les plus acceptables possibles du point de vue de la collectivité.

$\mathrm{Au}$ fil du temps, des réponses à ces deux catégories de préoccupation ont émergé. Dès les années 60, pour faire face à la première, on a eu recours (en France comme dans beaucoup d'autres pays développés, notamment anglo-saxons) aux outils de calcul économique du type de l'analyse coûts avantages. Ceux-ci amènent à calculer, comme dans le cadre de tout investissement qu'il soit public ou privé, des taux de rentabilité déterminés à partir d'une mise en balance des coûts et des avantages liés à un projet d'investissement. Ce calcul n'est pas sans poser un certain nombre de questions que nous allons soulever dans cet article.

En ce qui concerne la seconde catégorie de préoccupations, le législateur a tenté depuis plusieurs décennies d'institutionnaliser le débat entre le décideur public et les acteurs parties prenantes ${ }^{2}$. Cela nous amènera à soulever d'autres questions car d'une part l'appropriation par la loi de la problématique du débat public n'a été que très progressive et tardive et que, d'autre part, le débat mais également la contestation, voire le conflit, sont, comme le souligne $1^{\prime}$ 'encadré $\mathrm{n}^{\circ} 2$, ci-dessous, devenus des réalités dont il convient de tenir compte dans les processus de décision publique.

\begin{abstract}
Francis Beaucire écrit à propos des décisions susceptibles de mener à la réduction de l'usage de la voiture particulière pour des raisons environnementales ou économiques : « (...) dans ce domaine, une profonde transformation est intervenue : depuis une vingtaine d'années, dans la plupart des pays développés, il ne se prend plus aucune décision portant création d'un équipement ou d'une infrastructure sans un débat contradictoire entre les promoteurs et l'opinion publique, riveraine ou non, débat qui mène généralement au compromis, plus rarement au retrait du projet, encore assez souvent au désaveu électoral, et toujours moyennant une perte de temps sur les calendriers de réalisation que l'on peut interpréter comme le temps de la concertation. C'est-à-dire le temps de l'élaboration d'un consentement collectif. » (Expertise, débat public : vers une intelligence collective, coordonné par F. Goux-Baudiment, E. Heurgon et J. Landrieu, éd. de l'Aube)
\end{abstract}

Encadré $\mathrm{n}^{\circ} 2$ : l'évolution du contexte des décisions liées à la réduction de l'usage de la voiture particulière

On examinera dans cet article la façon dont s'articulent aujourd'hui en France les pratiques du calcul économique et du débat public. Tout d'abord, après avoir défini et présenté les principes de l'analyse coûts avantages (ACA) (cf. section 2), nous décrirons brièvement (cf. section 3) comment le débat public (expression qui ne signifie pas nécessairement débat «avec le public ») s'est institutionnalisé et à quel type de pratique cela a conduit. Ensuite, nous tenterons de mettre en perspective les difficultés liées à l'usage de l'ACA dans le cadre du débat public (cf. section 4) : celles-ci concernent, à notre avis, les exigences de rationalité et de légi-

\footnotetext{
2 Par 'acteur partie prenante' dans le cas de décisions portant sur un projet d'investissement en infrastructure de transport, nous entendons tout acteur détenteur d'un enjeu lié à la réalisation ou non du projet : par exemple une association d'usagers, une entreprise, une collectivité quelconque, etc.
} 
timité au sein des processus de décision liés aux infrastructures de transport. Nous terminerons en faisant quelques propositions susceptibles de faire évoluer ces pratiques afin que le déroulement du processus parvienne à mieux concilier la recherche conjointe des objectifs de rationalisation des choix d'investissements publics de transport et de légitimation des décisions correspondantes.

\section{Définition et principes de l'analyse coûts avantages}

Les pratiques des institutions représentatives de l'Etat, des collectivités locales ou territoriales en matière de choix d'investissement ont, pour la plupart, recours à des outils de calcul économique de type analyse coûts avantages. L'objet de cette section est de préciser le contenu de tels outils. Dans un premier temps, nous tenterons de mieux définir l'analyse coûts avantages en mettant en perspective notamment les concepts et éléments clefs de ce type d'analyse. Puis, dans un second temps, nous montrerons comment l'ACA est réalisée.

\subsection{Qu'est-ce que l'analyse coûts avantages?}

Après la période de forte croissance et de prospérité des trente glorieuses, les années 70 ont conduit les décideurs publics des pays occidentalisés à rationaliser l'utilisation des ressources budgétaires. La RCB (rationalisation des choix budgétaires) est une doctrine budgétaire dont la diffusion commence à cette époque (cf. Agard [1970]). Elle va influencer la plupart des pratiques de budgétisation qui s'appuient sur l'idée qu'il est possible de rationaliser au mieux les dépenses en vue d'atteindre des objectifs fixés à l'avance. Mais la RCB n'est pas uniquement et directement née de la crise des finances publiques. Elle provient surtout des critiques adressées dans les années 60 aux USA contre les pratiques traditionnelles à l'époque de construction et de suivi de budget. Manque de temps, manque de coordination entre départements de l'Etat et surtout manque de rationalité sont les arguments de la critique. Les USA vont apporter progressivement plusieurs réponses dont certaines sont inspirées des pratiques budgétaires du privé (celles de la Ford Motor Company entre autres). La RCB constitue une de ces réponses. Elle sera diffusée en France en tant que doctrine de budgétisation des choix publics à partir de 1968.

L'ACA, en tant qu'outil d'expertise et d'aide à la décision des décideurs publics, s'inscrit dans la lignée de la RCB (Le Duff et Papillon [1989]). L'ACA est une technique d'évaluation qui vise à éviter le gaspillage dans l'utilisation des ressources publiques. Elle conduit en théorie le décideur public à n'investir que dans les projets qui, d'un point de vue de la collectivité, sont les plus rentables possibles.

Dans ce but, elle doit être utilisée avant la décision pour évaluer, de façon comparative, les différents projets envisageables. Elle « vise à évaluer l'ensemble des effets directs et indirects d'un projet, ses effets financiers et non financiers sur l'ensemble des agents économiques concernés par l'investissement. Ces effets sont ensuite synthétisés, après monétarisation, dans un bilan socio-économique qui établit la rentabilité d'un projet, celle-ci étant appréciée sur la base d'indicateurs spécifiques » (Auzannet [1997]). Ainsi, l'ACA renvoie à plusieurs catégories d'enjeux d'ordre technique. Ceux-ci ont trait notamment à :

i) L'évaluation dans des unités appropriées d'un projet d'investissement sur toutes les dimensions, qu'elles soient ou non financières. Notons à titre illustratif, dans le cas de l'évaluation d'une infrastructure routière, la diversité des effets positifs à prendre en compte: gains de temps pour les utilisateurs du réseau de transport, 
économies en termes d'utilisation de la voiture particulière, gains en termes d'amélioration des conditions de sécurité des transports, économies de stationnement, gains en termes d'encombrement du réseau routier qu'emprunte la voiture particulière, économies d'entretien des voiries, impacts éventuellement positifs sur l'environnement.

ii) La monétarisation, c'est-à-dire l'évaluation en nombre d'unités monétaires, de ces effets très différents et parfois non marchands. Le Commissariat Général au Plan a conduit depuis plusieurs années des travaux qui ont abouti à préciser et à structurer sous forme de recommandations les méthodologies constitutives de l'ACA. Sur la question de la monétarisation des effets non marchands, le rapport du Commissariat Général au Plan de 2001 du groupe présidé par Marcel Boiteux (cf. CGP [2001]) est un ouvrage de référence. En introduction, M. Boiteux rappelle que la monétarisation «s'inscrit dans la préoccupation générale de valoriser les avantages et les inconvénients non marchands d'un projet, afin de fournir aux décideurs une évaluation complète des gains et des coûts engendrés par les diverses opérations et variantes entre lesquelles ils ont à choisir. ». La monétarisation des effets non marchands est donc un enjeu important et pose dans son opérationnalisation des questions majeures (cf. 2.2.1.).

iii) L'existence de plusieurs indicateurs synthétiques fournis par l'ACA, lesquels conduisent les décideurs publics à l'utiliser de différentes façons. L'ACA peut conduire à faire le calcul d'indicateurs de taux de rentabilité ou de bénéfices globaux que la collectivité peut potentiellement tirer d'un projet. Le calcul de chacun de ces indicateurs implique de définir :

- Une situation de référence en termes d'offre et de demande de transport. En effet, la monétarisation des avantages et des coûts porte sur l'évaluation de gains ou de pertes et donc sur le changement d'état d'un système. On montera (cf. 2.2.2.) que le choix de la situation de référence est très important car il a un impact direct sur les résultats en termes de rentabilité et de bénéfice.

- Une période de référence, c'est-à-dire une période sur laquelle porte l'analyse et l'actualisation. Ce choix est lui aussi déterminant et fait l'objet de travaux importants. Il faut, pour cette période de référence, être capable de construire un échéancier, c'est-à-dire un calendrier des valeurs monétaires des avantages et des coûts de l'investissement. L'introduction d'une longue période de temps implique de savoir également intégrer des effets de long terme tels que, par exemple, la variation du prix du pétrole ou encore la façon dont la pollution sera pénalisée dans les décennies à venir.

Ce qui précède montre que l'ACA est une technique complexe. Il nous faut maintenant examiner comment elle se pratique.

\subsection{Comment l'ACA est-elle réalisée?}

L'ACA applique, à une problématique de choix public, un raisonnement directement hérité de la théorie économique dominante sur la question des choix privés. Ainsi, le concept de rentabilité évoqué plus haut s'applique-t-il dans ce cadre aux décisions publiques puisque, comme dans les investissements privés, des ressources sont mises en regard de coûts et qu'il s'agit, 
comme pour les choix privés, d'optimiser l'utilisation de ces ressources nécessairement limitées. Toutefois, en raison de la complexité de l'analyse et des justifications de la démarche, le fonctionnement de l'ACA se heurte à des difficultés spécifiques tenant :

- aux procédures de monétarisation des éléments non marchands intégrés dans la balance des coûts et des avantages ;

- au choix de la période de référence et de la situation de référence pour pouvoir comparer un état projeté du système avec la situation qui prévaudrait en l'absence du projet, voire des projets concurrents en cours d'évaluation.

\subsubsection{La procédure de monétarisation des effets non marchands et effets externes}

Cette question est centrale car elle fonde la valeur attribuée à chacun des effets pris en compte dans le calcul de l'ACA. La procédure de monétarisation suit un schéma structuré en trois étapes distinctes.

La première étape consiste à identifier les avantages et les nuisances en des termes tels que les effets qu'il s'agit d'appréhender apparaissent comme une grandeur mesurable. Pour la grandeur ainsi définie, il faut expliciter une échelle physique de mesure. Il s'agit ensuite de concevoir et de mettre en œuvre une procédure appropriée pour mesurer, dans chacun des projets concernés, la quantité de la grandeur considérée qu'il conviendra de valoriser. Nous illustrons ci-après les difficultés de cette première étape avec les effets de la pollution atmosphérique.

La prise en compte de l'impact de la pollution atmosphérique liée aux transports, élément qui doit apparaître comme un coût dans le cas de l'étude par exemple d'un projet d'infrastructure routière, doit, avant d'être monétarisée, pouvoir être évaluée sous forme d'une grandeur physique mesurable. Ce n'est pas une question évidente souligne CGP [2001]. En effet, plusieurs éléments doivent être pris en considération :

- En premier lieu, la mesure de la pollution liée aux transports est intrinsèquement difficile. La pollution de l'air résulte de facteurs divers qui ne sont pas tous liés aux transports (chauffage, pollution industrielle, etc.) ; il convient ainsi de déterminer la part des émissions polluantes directement liées aux transports. De plus, la pollution concerne rarement une zone géographique bien délimitée ; elle peut être locale mais son impact est bien plus général, jusqu'à la planète entière, chaque émission polluante contribuant à l'effet de serre.

- En second lieu, l'impact de la pollution atmosphérique est nécessairement multiple (impacts sanitaires, impacts sur les capacités de production des entreprises, impacts sur les productions agricoles, impacts sur l'aménité des paysages, impacts olfactifs, etc.) et différentes procédures d'évaluation devraient donc être associées aux différents types d'impacts. En outre, la question particulière des relations entre doses de polluants atmosphériques et impact physiologique n'est pas tranchée.

En dépit de l'absence d'un consensus sur l'impact sanitaire en particulier, différents principes de calculs ont été retenus. 
1/ Le rapport indique tenir compte des progrès technologiques acquis et à venir. En effet, l'impact sanitaire de la pollution atmosphérique n'est pas aujourd'hui le même qu'hier en raison des progrès dans le domaine automobile notamment.

2/ Il faut également tenir compte de l'écart entre la ville et la rase campagne car l'impact d'une émission polluante n'est pas le même selon qu'elle survient en milieu où la population est très concentrée ou en milieu très peu urbanisé. Le rapport indique qu'il faut donc appliquer un coefficient correcteur à la valorisation de l'impact de la pollution liée à un projet d'investissement. Ce coefficient est différencié selon qu'il s'agit de prendre en compte l'impact de la pollution de véhicules légers ou de poids lourds mais également selon le type de terrain sur lequel est projeté l'infrastructure (cf. tableau ci-dessous).

\begin{tabular}{|c|c|c|c|}
\hline $\begin{array}{c}\text { Coefficient de cor- } \\
\text { rection }\end{array}$ & $\begin{array}{c}\text { Interurbain ou vallées } \\
\text { de montagne (plat) }\end{array}$ & $\begin{array}{c}\text { Interurbain ou vallées de } \\
\text { montagne accidentées } \\
\text { (pente assez faible 2 à 4 } \\
\%)\end{array}$ & $\begin{array}{c}\text { Interurbain ou vallées de } \\
\text { montagne très acciden- } \\
\text { tées (pente assez faible 4 } \\
\text { à 6\%) }\end{array}$ \\
\hline Véhicules légers & 1,1 & 1,1 & 1,1 \\
\hline Poids lourds & 0,95 & 1,5 & 2,1 \\
\hline
\end{tabular}

Tableau $\mathrm{n}^{\circ} 1$ : Coefficient correcteur pour la prise en compte de l'impact de la pollution atmosphérique (Source CGP [1994])

La deuxième étape débute par l'inventaire des procédures appropriées pour attribuer une valeur monétaire à chacune des unités physiques retenues dans le cadre de la première étape. Ces procédures peuvent faire intervenir des enquêtes dites de préférences déclarées ou d'évaluations contingentes mettant en évidence des consentements à payer ou à recevoir. Elles peuvent aussi faire référence à des marchés existants permettant d'apprécier indirectement l'impact de l'avantage ou de la nuisance, l'observation de comportements qu'adoptent effectivement des individus étant susceptible de révéler leurs préférences. La monétarisation du bruit est un exemple de procédure qui s'appuie sur la référence à un marché existant. Il s'agit du marché immobilier. Des études ont montré, dixit CGP [2001], des relations significatives entre des niveaux d'exposition au bruit et la dépréciation de valeurs immobilières (cf. tableau suivant).

\begin{tabular}{|c|c|c|c|c|c|}
\hline $\begin{array}{c}\text { Niveau de bruit } \\
\text { en façade (en } \\
\mathrm{dB})\end{array}$ & 55 à 60 & 60 à 65 & 65 à 70 & 70 à 75 & $\begin{array}{c}\text { Au delà de } \\
75\end{array}$ \\
\hline $\begin{array}{c}\% \text { de déprécia- } \\
\text { tion/décibel }\end{array}$ & 0,4 & 0,8 & 0,9 & 1 & 1,1 \\
\hline
\end{tabular}

Tableau $\mathrm{n}^{\circ} 2$ : Coefficient de dépréciation immobilière en fonction du bruit en façade (Source : CGP [2001])

CGP [2001] recommande de monétariser le bruit en prenant en compte cette dépréciation appliquée sur le prix moyen des logements locatifs ; le rapport précise : " le coût unitaire du bruit est défini par la dépréciation des prix moyens de location par $\mathrm{m} 2$ de surface occupée et exposées à des niveaux de bruit dépassant un seuil. Loyer mensuel au $\mathrm{m} 2$ locatif publié par l'INSEE à l'échelle nationale » (chapitre 8 du rapport).

Enfin, lors de la troisième étape de la procédure de monétarisation, il s'agit de déterminer avec précision les évolutions dans le futur d'un certain nombre de valeurs de référence retenues dans le calcul de l'ACA. Il faut donc ici faire de la prévision, activité qui conduit à pronostiquer ce que seront dans le futur les diverses valeurs de référence et notamment celles qui 
concernent la pollution atmosphérique, le bruit ou encore la vie humaine épargnée. A titre d'exemple, notons que CGP [2001] recommande de considérer que la pollution atmosphérique liée aux transport se réduit année après année à un rythme de $6,5 \%$ par an pour les poids lourds et de 9,4\% par an pour les véhicules particuliers et les véhicules utilitaires légers. La valeur de la vie humaine épargnée est considérée comme devant croître à un rythme équivalent à celui de la consommation des ménages par tête.

\subsubsection{Utilisation des valeurs monétaires, calculs de rentabilité des projets et situation de ré- férence}

Les chiffrages monétaires précisés ci-dessus servent ensuite de référent pour l'évaluation sous forme d'ACA de tout type de projet d'infrastructure. Pour évaluer un projet d'investissement, l'ACA prend en compte l'ensemble des changements en termes d'avantages ou de coûts pour la collectivité induits par le projet sous forme de grandeurs physiques, autrement dit tous les effets directs et indirects (tous ceux qui impliquent dépenses et recettes monétaires mais également le bruit, la pollution, la sécurité, les conditions de vie, les impacts sur les générations futures, etc.) puis les monétarise à l'aide des valeurs de référence.

L'évaluation des gains et des coûts procurés par un projet d'investissement nécessite de caractériser avec précision la situation de référence. Il est en effet indispensable de faire intervenir une telle situation puisque les avantages et les coûts à prendre en compte sont en fait des écarts qu'il s'agit d'apprécier au travers des changements qu'occasionnera le projet s'il est mis à exécution. Cette situation est donc définie comme étant celle qui prévaudrait en l'absence du projet ou des projets concurrents. Par exemple, les gains en termes de décongestion associés à un projet d'infrastructure routière ne peuvent être appréciés que si les trafics présents et surtout leur évolution dans le futur en l'absence de cette infrastructure peuvent être eux-mêmes appréciés. CGP [1994] précise que la situation de référence n'est que très rarement une situation de statu quo, c'est-à-dire une situation où l'on ne fait rien. En effet, si l'on ne réalise pas le projet considéré, il faut alors opérer des investissements nécessaires aussi bien pour maintenir l'offre de transport à un même niveau qualitatif et quantitatif que pour entretenir les infrastructures de transport existantes. La caractérisation d'une situation de référence dans une démarche d'ACA nécessite donc la prise en compte de tels investissements dits « éludés » car non effectués si le projet est réalisé. A l'inverse, la situation de référence peut correspondre à une situation qui se dégrade en raison précisément de la non réalisation du projet d'investissement évalué. Dans tous les cas, l'évaluation des avantages et des coûts d'un projet n'est possible que si les coûts des investissements éludés ou la dégradation en tous points de vue de la situation qui surviendrait si l'on ne réalisait pas l'investissement sont intégrés dans la situation de référence avec précision de façon à ce que cela soit pris en compte dans les calculs de rentabilité associés. La caractérisation de la situation de référence est un exercice fondamental et délicat puisque « dans de nombreux cas c'est dans sa définition que réside la principale source d'incertitude sur la rentabilité des projets (niveau des trafics de référence par exemple) » (CGP 1994). Par ailleurs, il est parfois difficile de clairement distinguer d'un côté la situation de référence et de l'autre côté la situation avec réalisation du projet d'investissement. En effet, il est évidemment tout à fait envisageable de considérer l'évaluation de variantes intermédiaires, évaluation qui rend moins évidente la définition de ce qu'est précisément la situation de référence. Enfin, l'évaluation de la situation de référence n'est pas entièrement détachée de la réalisation d'autres projets d'investissement déjà évalués et qui ont une probabilité de survenance forte. Dans ces circonstances, il peut être nécessaire de produire plusieurs situations de référence. Ceci risque de faire perdre de leur signifiance et de leur portée aux calculs de rentabilité qui suivent. 
Les calculs de rentabilité constituent également une étape importante des démarches d'ACA. Ils peuvent prendre la forme de deux types d'indicateurs :

1/ le taux de rentabilité immédiate défini comme le rapport pour la première année de mise en service de l'infrastructure de transport entre les avantages évalués et monétarisés pour la collectivité et le montant total de l'investissement ;

2/ le taux de rentabilité interne qui mesure le taux d'actualisation pour lequel la somme actualisée des avantages monétarisés égale la somme actualisée des coûts du projet d'investissement.

L'ACA peut encore conduire au calcul d'un bénéfice actualisé ou valeur actuelle nette qui prend en compte tous les coûts et avantages sur un horizon temporel défini par avance. Le calcul de cette valeur nécessite de définir un taux d'actualisation. Pour donner de la pertinence et de la signifiance aux calculs réalisés à partir de ce taux, des recommandations ont été faites par le Commissariat Général au Plan de sorte que les pratiques des administrations et des institutions représentatives de l'état soient unifiées ${ }^{3}$.

Plus généralement, la pratique des démarches d'ACA oblige à définir de nombreuses valeurs de référence et à expliciter un grand nombre d'hypothèses, donnant ainsi l'image de procédures très techniques et très formalisées. Ceci nous conduit, dans la prochaine section, à questionner l'intégration de telles pratiques au sein de phases de débat public, d'autant que, parallèlement, le débat public en tant que pratique de la décision publique en matière d'infrastructure de transport s'institutionnalise très progressivement et avec difficulté.

\section{Législation, outils et pratique du débat public}

Le débat public nécessite d'être structuré et organisé avec rigueur pour pouvoir en tirer avantage. En France, notamment dans le cas des décisions portant sur l'environnement et les infrastructures de transport, le débat public a tardé à s'instituer et tarde encore à se généraliser en tant que pratique de la décision publique. Sa prise en compte effective dans la loi est relativement récente. C'est ce que nous tenterons de mettre en évidence dans un premier temps. Par ailleurs, la loi a institué différents outils pour la pratique du débat public, outils qui ne servent pas véritablement à promouvoir le débat public. C'est ce que nous verrons dans un second temps en nous appuyant sur deux de ces outils qui nous paraissent intéressants : la procédure d'enquête publique préalable et la Commission Nationale du Débat Public (CNDP). Enfin, dans un troisième temps, nous nous appuierons sur le cas édifiant du troisième aéroport parisien pour montrer que la difficulté pour le débat public à s'intégrer dans le déroulement du processus de décision publique provient en grande partie d'un déficit d'expertise général de la démocratie participative.

\subsection{Ce que la loi dit à propos du débat public}

Dans les faits, la remise en cause des choix publics a mis en évidence depuis longtemps avant même que celle-ci soit sujette à médiatisation accrue - les faiblesses du système de démocratie représentative et ce dans le cas notamment des décisions relatives à l'environnement et aux infrastructures de transport.

\footnotetext{
${ }^{3}$ Le taux préconisé par le Commissariat Général au Plan est actuellement de $8 \%$ (valeur à nouveau en discussion)
} 
L'apparition de dispositions légales relatives à l'organisation d'un débat public caractérisant une démocratie des décisions publiques plus participative est finalement relativement récente. Elle résulte d'un processus historique progressif dont on peut identifier trois temps forts (cf. Plottu [1998]).

Le premier temps est celui de la décennie 60-70. Sans aller jusqu'à institutionnaliser un débat public, il marque la prise en compte par la loi de nouvelles sensibilités des citoyens. La loi du 10 juillet 1976 en particulier est importante car c'est elle qui institue, dans le cas des décisions relatives aux infrastructures lourdes de transport, la réalisation d'études d'impacts sur l'environnement et ceci est tout à fait nouveau à l'époque. Néanmoins, cette prise en compte est toute relative et, dans la pratique, ce sont les études de rentabilité qui prédominent. Les décideurs publics visent clairement des projets qui minimisent les coûts directs et ceux qui améliorent les conséquences directes pour l'usager. Dans tous les cas, la place laissée au débat public est nulle. « Rien dans l'enquête publique n'est vraiment adapté : publicité, délais, choix du commissaire enquêteur, présentation du dossier ne permettent aucunement de peser sur la décision. Entre l'acceptation passive et le refus militant il n'y a pas de place pour une recherche démocratique d'une meilleure solution » (cf. Flaque [1987] dans Plottu [1998]).

Le deuxième temps, celui de la décennie 80, est marqué par la Loi d'Orientation sur les Transports Intérieurs (LOTI) du 30 décembre 1982 qui est probablement le texte le plus important pour les décisions publiques de transport. Aujourd'hui encore, ce texte régit un grand nombre de décisions de transport public en France pour la plupart des régions (la région Ilede-France n'y est cependant pas soumise). Sur la question du débat public, la LOTI a surtout contribué à un peu plus de transparence des décisions. Elle institue un dossier d'évaluation économique et social, sorte de document de référence qui suit un projet tout le long des examens et des études qui jalonnent le processus de décision. Trois types d'études sont précisément associés aux projets d'infrastructure et de transport : l'étude technique, l'étude de trafic (ou étude socio-économique) et l'étude d'environnement. Malgré tout, durant la décennie 80, ces trois examens sont juxtaposés sans réelle vision conjointe des points de vue multiples. Il y a bien la loi Bouchardeau du 12 juillet 1983 qui parle de participation du public au processus de décision mais le débat en question ne vient que bien après les études techniques qui ont déjà, dans la pratique, validé un projet.

La décennie 90 correspond au troisième de ces trois temps forts. Celui-ci a été marqué par de profondes réflexions sur la question de la concertation et du débat avec les acteurs parties prenantes dans les décisions publiques. Le rapport Carrère de 1992 préconise ainsi que les processus de décision publique en matière d'infrastructure et de transport soient construits sur la base d'une concertation située en amont réel de la décision. Cette préconisation est reprise dans les textes de loi qui suivent. La circulaire Bianco (1992) impose une phase de débat en amont des études de tracés d'infrastructure. Dans la circulaire du 27 septembre 1993, nous lisons à propos des objectifs des études d'impact : « aider, (...), éclairer le décideur sur la nature et le contenu de la décision à prendre, informer le public pour lui donner les moyens de jouer son rôle de citoyen averti et vigilant». Enfin, la loi Barnier du 2 février 1995 sur les «Dispositions relatives à la participation du public et des associations en matière d'environnement » institue une commission nationale du débat public qui est en charge de l'organisation des débats avec l'objectif sous-jacent de veiller à l'organisation de la concertation en amont. Certains auteurs indiquent avec optimisme que l'institution de cette commission marque le début d'une nouvelle ère de la décision publique (cf. Blanc [1997]). Néanmoins, ces mêmes auteurs attirent également l'attention sur les difficultés rencontrées pour définir clairement la phase amont d'un processus de décision. 
La loi a malgré tout cherché à institutionnaliser le débat grâce à quelques outils aménagés pour faire intervenir certaines des parties prenantes dans le cours du processus de décision. Tout récemment, l'instruction cadre du 25 mars 2004 (cf. DTT [2004]) a mis à jour un certain nombre de textes de lois relatifs au transport intérieur. Ce texte est désigné par le législateur comme devant servir de référent. Il précise en outre certaines dispositions relatives (et c'est ce point qui, à plusieurs reprises, retiendra ensuite notre attention) à la façon d'articuler les phases d'évaluation socio-économique des projets et les phases de débat public.

\subsection{Institutions et outils du débat public à l'heure actuelle en France}

Nous présentons ci-après les deux outils qui nous semblent être les plus représentatifs de l'évolution des deux dernières décennies. Il s'agit de la procédure d'enquête publique et de la commission nationale du débat public.

\subsubsection{La procédure d'enquête publique}

La procédure d'enquête publique est ancienne puisque c'est une loi de 1833 qui l'institue. Au départ, cette enquête n'a que peu de motivation démocratique. Elle est juste un moyen accordé aux propriétaires fonciers pour protéger leur droit de propriété privée. Elle ne concerne ainsi que les cas d'expropriation. Pendant très longtemps, l'enquête publique reste le seul apanage des propriétaires terriens. Il faudra attendre les années 70 et 80 pour qu'elle devienne un instrument au service des associations de défense de l'environnement. La loi Bouchardeau de 1983 institue, pour chaque projet qui est susceptible d'avoir un impact non négligeable sur l'environnement, une enquête préalable et publique systématique. Ainsi, la population concernée et, plus généralement, l'ensemble des parties prenantes ont la possibilité de venir consulter les études d'impact et toutes les informations relatives à un projet qui porte atteinte à l'environnement. Un commissaire enquêteur, indépendant et nommé par un tribunal, recueille ensuite les avis, suggestions et contre propositions. Néanmoins, l'enquête publique ne constitue pas réellement un outil de démocratie participative et est soumise à une critique forte (cf. Blanc [1998]). En premier lieu, elle intervient certes en amont des décisions mais à un stade déjà bien avancé où les projets sont définis de telle sorte que seules des modifications très marginales peuvent être envisagées. De ce fait, les enquêtes publiques ressemblent plus à des outils de consultation et à des outils destinés à convaincre une population de parties prenantes qu'à un projet fondamentalement bon. N'est-il pas paradoxal de lire (cf. Molines [2003]) que, pour être recevable, la critique d'un projet « doit être émise par une personne directement concernée par le projet mais ne doit pas être liée à la défense d'intérêt particulier ». Ainsi, les oppositions aux projets ne peuvent être que brutales et conduisent généralement à des situations de blocages très loin des objectifs véritablement recherchés dans une vraie concertation. Enfin, l'information à disposition du public est d'une très haute technicité. Le public néophyte est ainsi conduit à devenir suspicieux à l'égard de la procédure dont il sent qu'il est de fait écarté.

\subsubsection{La Commission Nationale du Débat Public}

La commission nationale du débat public a été instituée par la loi Barnier de 1995. Elle constitue aujourd'hui un outil juridique et, surtout, institutionnel privilégié pour les questions de débat public. Les projets qui présentent un enjeu et des impacts potentiels forts sur l'environnement doivent impérativement faire l'objet d'un débat en amont. La CNDP est précisément la commission garante de la tenue correcte de ce débat public. Les modalités d'intervention de cette institution sont diverses. Elle peut intervenir sur demande d'un minis- 
tère pour prendre en charge l'organisation d'un débat public autour de projets. Elle peut être saisie par plusieurs parlementaires (20 au moins) ou par les conseils régionaux du ou des territoire(s) concerné(s) par le projet. Dans ce cas, la CNDP est chargée de vérifier que le ou les projet(s) est (sont) bien conformes à la loi. Enfin, dans certains cas, elle peut être saisie par des associations de protection de l'environnement agréées. Dans ce dernier cas, la commission est libre de donner suite ou non à la saisine. Dans le cas où elle décide de donner une suite favorable, la CNDP instruit le dossier et constitue pour chaque projet une commission chargée de l'instruction. La CNDP n'est pas un arbitre qui tranche sur le fond du dossier. Il s'agit uniquement d'une institution en charge de la tenue de la phase de débat public ou de la vérification de la bonne tenue du débat public dans le cas où ce n'est pas elle qui en assure l'organisation (et dans le cas où elle fait l'objet d'une saisine).

\subsection{Une expérience de débat public : le troisième aéroport parisien}

Les difficultés d'institutionnalisation du débat public reflètent certainement plus une maladresse généralisée et un manque d'expertise des décideurs publics en la matière qu'une volonté de museler à tout prix l'opinion publique. Le cas du troisième aéroport parisien est à ce titre tout à fait édifiant.

Les pouvoirs publics ont lancé, au début de l'année 2001, une démarche qui devait être fondée sur la " démocratie de proximité » participative. Un débat public sur la question du choix du site du troisième aéroport parisien fut donc organisé avec l'originalité de se situer nettement plus en amont que cela n'était le cas dans la plupart des processus de choix d'investissement public. Le débat a été confié à la commission DUCSAI (Démarche d'Utilité Concertée pour un Site Aéroportuaire International). Il avait pour objectif de faire émerger différentes alternatives possibles. L'état a annoncé qu'aucune alternative n'avait été préalablement évaluée de sorte que la démarche s'apparentait réellement à de la concertation. En fait, beaucoup d'observateurs ont montré que cette phase du processus avait fait apparaître un grand nombre d'ambiguïtés (cf. Fourniau [2002]).

En premier lieu, les engagements de l'état étaient peu clairs. L'état s'est engagé en 1997 à plafonner le trafic sur les aéroports existants de Roissy et d'Orly (Discours du Ministre des Transports J.C. Gayssot de 1997). Un troisième aéroport parisien devait permettre de désengorger ces deux sites aéroportuaires et ainsi de respecter les limites de trafic fixées par l'état. Néanmoins, les experts ont indiqué à l'époque que ces limites seraient atteintes très largement avant que le troisième aéroport ne soit en fonction. Les engagements de l'état ne pouvaient donc raisonnablement pas être tenus.

En deuxième lieu, la direction de l'aviation civile avait préalablement au débat, préparé trois propositions de sites alors que, comme précisé plus haut, l'état s'était engagé à ne préjuger d'aucun projet avant le débat de la DUCSAI. Par ailleurs, les collectivités et les acteurs économiques locaux ont proposé des candidatures pour une question qui, évidemment, était d'importance nationale. Ceci conduit donc à s'interroger sur la légitimité des dossiers de candidature.

En troisième lieu, la maîtrise d'ouvrage assurée par la direction de l'aviation civile n'a finalement pas tenu compte des questions soulevées par le débat et s'en est tenue au seul critère de ses prévisions de trafic. 
Enfin, en dernier lieu, l'auteur note que le rôle de la commission DUCSAI dans le débat était lui-même ambigu. Pour preuve, le président de cette commission assurait également la fonction de président de la commission nationale du débat public, institution en cours de réforme à l'époque.

En outre et bien que d'apparence très souple et très en amont du processus, la DUCSAI avait une durée limitée à six mois. La faiblesse des données disponibles pour mener un débat structuré et argumenté imposait théoriquement que des études préalables soient faites, ce qui n'a pas été le cas (cf. Barraqué [2002]). De plus, l'hypothèse de départ de l'ensemble du débat, à savoir l'utilité même d'un troisième aéroport parisien, n'a pas pu être remise en question en dépit des demandes faites (par faute de temps ?). Après l'attentat du 11 septembre 2001, les trafics de voyageurs ont considérablement chuté et ont remis en cause de fait l'utilité du troisième aéroport.

Il est ainsi possible de défendre l'idée que la DUCSAI n'a pas été un instrument de concertation visant à la prise en compte des points de vue de tous. Elle fut entendue comme un instrument de légitimation de choix qui appartiennent aux autorités publiques (cf. Lascoumes [2002]).

\section{Pratique et valeur des démarches d'ACA, contributions au débat public}

Le cas du troisième aéroport parisien montre qu'au delà des difficultés de mettre en œuvre des instruments ou des institutions dédiés à un débat public systématique, la décision publique se heurte à une difficile déconstruction des routines et des rationalités qui structurent la façon de prendre les décisions relatives aux infrastructures de transport. Il est intéressant de noter d'ailleurs qu'aucune démarche d'ACA - démarche de fait très largement associée à la décision publique telle qu'elle est traditionnellement pratiquée - ne semble avoir été conduite dans le cadre de la DUCSAI. L'absence de recours à l'ACA dans le cadre d'une démarche de débat public pose question. En effet, si l'ACA consiste, comme nous l'avons vu, en une prise en compte de tous les avantages et de tous les coûts d'un projet d'investissement pour la collectivité en vue d'une utilisation la meilleure possible des deniers publics, alors pourquoi l'ACA n'a t-elle pas instrumenté la DUCSAI qui visait précisément à satisfaire au mieux la collectivité et à dégager un choix de site aéroportuaire le plus acceptable possible ? Cette absence nous invite à questionner l'articulation possible entre ACA et pratique du débat public. Avant de le faire (cf. 4.2.) nous souhaitons mettre en évidence les limites de l'ACA pour garantir la meilleure utilisation des ressources publiques. Ces limites font apparaître l'ACA comme un instrument conventionnel pour asseoir des évaluations. Celles-ci, pourvu qu'on les regarde comme le résultat de conventions, peuvent jouer un rôle fort utile dans le déroulement du processus de décision. 


\subsection{Limites de l'ACA au service de la rationalisation des choix publics}

La démarche d'ACA vise à identifier les investissements en infrastructures qui, du point de vue de la collectivité, sont les plus rentables possibles. Cette démarche est souvent perçue comme étant apte à découvrir par des voies scientifiques des éléments objectifs permettant de justifier la réalisation ou l'abandon de projets d'investissements. Cette aptitude est en fait limitée (cf. 4.1.1. et 4.1.2.); la présence de biais dans la mise en œuvre de l'ACA (cf. 4.1.3.) vient encore réduire cette aptitude.

\subsubsection{Objectivité des démarches d'ACA}

Un des aspects les plus importants de l'ACA est d'intégrer, dans un calcul de rentabilité, des éléments de gain ou de coût correspondant à des phénomènes qui n'existent pas dans la réalité sous forme monétaire ou quantitative. Cette intégration s'opère à l'aide de nombreux équivalents monétaires tels que la valeur de la vie épargnée (dont on peut se demander s'il est justifié d'adopter des valeurs différentes pour les transports en commun et les transports individuels), la valeur du temps gagné (dont on peut se demander selon quels facteurs il convient de la moduler : zone géographique, profession et catégorie sociale, motif de déplacement, ...), la valeur du taux d'actualisation (la préférence pour le présent ne dépend-elle pas des biens considérés ?), la valeur du bruit, etc. Ceci conduit à faire intervenir des valeurs dites tutélaires. Leur caractère objectif prête souvent à discussion: elles correspondent à des construits et non à des réalités directement chiffrables en unités monétaires. On peut donc penser qu'elles contiennent une grande part d'arbitraire et, parfois même, de prises de positions politiques. Il convient donc de relativiser l'objectivité des calculs de rentabilité effectués sur de telles bases.

L'ACA fait porter ces calculs sur de longues périodes en accordant un poids très faible au futur lointain. Notre appréhension du futur est cependant toujours sujette à d'importantes controverses. En particulier, l'évaluation des gains de temps reposent sur des modèles de prévisions de trafic à propos desquels on lit, dans CGP [2001], qu'ils « fournissent une grande diversité de résultats, (...) En outre les erreurs de prévisions de trafic sont élevées et peuvent atteindre 10 à $20 \%$ du trafic total sur une infrastructure donnée. On connaît de nombreux cas où l'erreur est d'un ordre de grandeur bien supérieur. ». Sur le même sujet, Cahen et Colombo [1999] écrivent : «Les modèles de trafic ne sont pas probants. Tout au plus peuvent-ils délivrer des résultats non invraisemblables. Les modèles ne servent pas à démontrer ou affirmer un résultat mais constituent des études plaidoyer ». L'objectivité est donc toute relative, d'autant plus que les mutations sociales et les évolutions des comportements sont constantes.

Il s'ensuit que les valeurs retenues pour les calculs ne sont toujours que des valeurs de convenance qui présentent une part d'arbitraire souvent importante : voir les variations dans le temps et entre les pays européens des valeurs de la vie humaine, du temps, de l'occupation des sols ou encore du taux d'actualisation. En dépit de cela, ces valeurs sont souvent présentées, sinon comme exactes, du moins comme une bonne approximation d'une réalité objective. Jean-Michel Charpin, dans l'avant-propos de CGP [2001], écrit d'ailleurs : " Il s'agissait de se mettre d'accord sur les valeurs monétaires à attribuer à des phénomènes rebelles au chiffrage : impact du bruit des trafics sur la santé, effets nocifs de la pollution atmosphérique, vies humaines épargnées, temps gagné (...) Les estimations obtenues sont inévitablement imparfaites. Elles prêtent donc à controverse. ». Une telle position sous-entend qu'il existe quelque part des valeurs exactes qu'il s'agit d'approcher. L'expression 'valeur révélée' est significative de cette conception: "Savoir comment révéler le consentement à payer pour l'environnement dans le secteur des transports » est la problématique qui guide bon nombre 
de ceux qui cherchent à attribuer une valeur à ces coefficients de pondération (Andan et al. [1995]).

Pourtant, l'imperfection des mesures ne saurait être attribuée au seul manque d'information. Elle tient aussi à l'impossibilité de saisir des valeurs intrinsèques qui seraient sensées exister dans notre société à une époque donnée, valeurs qui seraient indépendantes du modèle et du mode d'appréhension : les biens non marchands ont-ils véritablement une valeur immanente dans notre société que l'ACA ne ferait que révéler? Les multiples raffinements préconisés au fil des différents chapitres de CGP [2001] montrent bien qu'il s'agit beaucoup plus de construire des normes plutôt que d'approcher des valeurs existantes.

\subsubsection{Scientificité des démarches d'ACA}

Le caractère scientifique et neutre des démarches d'ACA est sensé leur conférer une légitimité arbitrale sur la question des choix publics en matière d'investissements en infrastructures de transport en particulier. La scientificité de l'ACA se justifie si l'on accepte les hypothèses qui fondent plus généralement le calcul économique du paradigme de l'orthodoxie libérale. DTT [2004] rappelle ainsi que le «bilan économique est le meilleur critère d'appréciation de l'efficacité socio-économique d'une décision dans des conditions d'optimalité que la théorie a précisées, qui ne sont certes jamais réunies dans la réalité, mais qui en donnent une approximation rationnelle. ». En particulier, il faut accepter de considérer que la collectivité n'a qu'un seul objectif, celui de la maximisation d'un surplus collectif. La collectivité est considérée comme un bloc monolithique car constituée d'individus tous identiques et parfaitement rationnels que la théorie économique désigne sous l'appellation 'homo oeconomicus'. Il s'agit de la modélisation d'un individu représentatif capable d'optimisation et capable de faire les choix optimaux en termes d'allocation de ses ressources rares à usage de satisfaction de ses besoins. Dans le cas d'un investissement public, l'objectif de maximisation du surplus collectif n'a de valeur que si - et c'est ce que la théorie considère - les gains que représente cet investissement pour certains sont redistribués vers les individus pour qui il représente un coût.

De plus, le programme rationnel et optimisateur du calcul économique de l'homo oeconomicus ne nous semble réalisable que si d'une part un optimum existe réellement et si, d'autre part, la démarche de calcul mise en œuvre conduit effectivement à déterminer ce qui est meilleur et ce qui est moins bon ; cela peut se traduire concrètement de la façon suivante pour les démarches d'ACA : l'ACA permet de déterminer les projets les meilleurs pour la collectivité si, sur la base d'une étude exhaustive (et totalement dégagée de tout parti pris), des avantages et des coûts pour la collectivité de tout projet d'investissement, ceux qui font apparaître un bénéfice net actualisé le meilleur possible, émergent. L'exigence d'exhaustivité nous paraît difficilement tenable. Le législateur a lui même concédé que des éléments non pris en compte dans l'ACA pouvaient intervenir dans la prise de décision à côté du bilan socio-économique que permet de réaliser l'ACA (cf. DTT [2004]). D'autres arguments mettant en question la scientificité des démarches d'ACA peuvent être avancés. Nous les avons discutés dans un autre article (cf. Roy et Damart [2002]) et ne les reprendrons pas ici.

\subsubsection{Biais dans la pratique de l'ACA}

La mise en œuvre des démarches d'ACA n'est pas exempte de biais importants qui peuvent fausser les choix qui découlent de l'évaluation socio-économique des projets d'investissements. Le choix de la situation de référence par exemple est évidemment sujet à biais. La détermination de la situation de référence (cf. 2.2.2.) est un exercice qui laisse une 
grande liberté d'appréciation susceptible d'être utilisée de façon partisane. En effet, l'évaluateur doit, comme nous l'avons souligné, faire intervenir des hypothèses pas toujours faciles à expliciter bien que leur impact puisse être important. C'est en particulier le cas des investissements qu'il ne sera pas nécessaire de réaliser si le projet évalué est retenu. Il doit également tenir compte des autres investissements déjà prévus et qui auront un impact sur la situation de référence, modifiant probablement par exemple les trafics prévisibles, les transferts de déplacements de population depuis un mode de transport vers un autre.

Dans la mesure où l'ACA peut jouer un rôle privilégié pour orienter les choix stratégiques que fait un pays pour ses infrastructures de transport, les biais qu'elle peut comporter sont loin d'être négligeables. Pour le montrer, nous prenons ci-après appui sur le cas des autoroutes ferroviaires (cf. Hammiche et Denant-Boemont [1997]).

Depuis plusieurs décennies, le trafic routier ne cesse de s'intensifier. En 2010, les principales artères autoroutières et routières seront totalement saturées et, par ailleurs, le trafic des camions de transports de marchandises constituera une part non négligeable de l'ensemble des flux. Par ailleurs, la SNCF souhaite depuis longtemps renforcer sa position concurrentielle sur le marché du transport de marchandises. La solution de l'autoroute ferroviaire consiste à faire circuler, sur les longues distances, les camions de transports de marchandises (selon les versions le tracteur et la remorque ou la remorque seule) sur des trains adaptés. Ce système de transport se situe en fait à mi-chemin entre la route et le train. Dans le cas où tracteur et remorque sont transportés ensemble, il évite les ruptures de charges car l'acheminement des marchandises vers les points où le train ne peut aller et qui restent dédiés au camion est facilité. Par ailleurs, le fait de transporter un poids mort (le tracteur) est fortement compensé par le fait que la vitesse commerciale de transport est sensiblement plus importante que celle du camion : elle est de $120 \mathrm{Km} / \mathrm{h}$ selon les auteurs de l'article cité plus haut. Ensuite, la vitesse de déchargement/chargement est réduite grâce à des solutions techniques innovantes. Si c'est le train qui assure le transport, l'autoroute ferroviaire est néanmoins relativement plus proche du concept d'autoroute. En effet, les autoroutes ferroviaires sont reliées aux autoroutes par les nœuds d'interconnexion qui ressemblent à ceux liant les autoroutes aux routes secondaires.

L'examen des ACA réalisées sur de tels projets montre, selon les auteurs, certains des biais de cette démarche qui a conduit systématiquement à surévaluer la rentabilité des projets d'infrastructure routière et, inversement, à sous-évaluer celle des projets d'autoroute ferroviaire. Ces biais sont de plusieurs natures. Ils concernent les effets externes du transport par la route et les hypothèses sur la tarification des transports par route et par autoroute ferroviaire et ses implications en termes de transfert modal.

En premier lieu, l'évaluation des projets d'autoroute ferroviaire ne prend pas en compte certains effets susceptibles pourtant d'accroître la rentabilité de ces projets. Les auteurs évoquent notamment les effets négatifs du transports de marchandises par la route, évités par le recours à l'autoroute ferroviaire et qu'il faut comptabiliser en gains.

En second lieu, les calculs de rentabilité dans les deux cas s'appuient sur des hypothèses de tarification du transport structurellement défavorables à l'autoroute ferroviaire. En effet, l'évaluation de l'autoroute ferroviaire envisage un prix au kilomètre de l'ordre de $3 \mathrm{~F}(0,45 €)$. L'élévation de ce prix permettrait dans les calculs d'augmenter sensiblement la rentabilité du ferroviaire. Néanmoins, dans la réalité, un prix plus élevé rendrait la route beaucoup trop attractive pour espérer que l'autoroute ferroviaire puisse être concurrentielle. Ensuite, l'Etat a systématiquement encouragé le transport des marchandises par la route à travers une politique 
fiscale fortement incitative, se traduisant notamment par la non revalorisation de la taxe à l'essieu depuis 1971. Par ailleurs, l'extrême intensité concurrentielle dans le secteur a conduit beaucoup de transporteurs à fournir du service de transport de marchandises à un prix qui ne permet pas d'atteindre le seuil de rentabilité. Autrement dit, la comparaison des rentabilités des projets « route» et «autoroute ferroviaire» est fortement biaisée : les tarifs de la route pris en compte dans le calcul sont inférieurs à ce qu'ils devraient être pour assurer la rentabilité du service d'une part, l'un des deux projets mis en balance est fiscalement favorisé par l'état, aide qui en biaise le calcul de rentabilité d'autre part.

Les considérations présentées depuis le début de cette quatrième section montrent que l'ACA ne peut permettre à elle seule de déterminer les choix d'investissements les meilleurs possibles pour la collectivité. Au delà, malgré les biais évoqués plus haut, 1'ACA est-elle porteuse d'une rationalité qui suffit à rendre légitimes et acceptables les choix publics en matière d'investissements en infrastructures de transport? Autrement dit, la pratique de l'ACA peutelle servir un débat public dont le fondement est précisément la recherche des investissements les plus acceptables et légitimes possibles ? Ci-après, nous tentons d'apporter des éléments de réponses à ces questions.

\subsection{Calcul économique et débat public}

La pratique du débat public a pour fondement la recherche, avec les acteurs parties prenantes, des choix les plus acceptables pour la collectivité sans passer par le biais d'un système représentatif en quête de légitimité.

La pratique du débat public constitue un mode de gouvernance qui, en France, s'implémente avec difficulté comme nous l'avons vu à propos du troisième aéroport parisien (cf. 3.3.). La nécessité d'y recourir se justifie en partie par les insuffisances de l'ACA à déterminer des choix d'investissement conformes à un intérêt général. D'un autre côté, les risques liés à l'instauration de phases de débat public nécessairement difficilement structurables et formalisables réhabilitent-ils des démarches structurées fondées sur le calcul économique ?

Faire participer de multiples acteurs dits parties prenantes, c'est se heurter aux difficultés inhérentes à la structuration de la participation d'acteurs multiples à un processus qui doit nécessairement être cadré pour éviter toute dérive de type " foire d'empoigne ». Les démarches d'ACA ont l'avantage de constituer un cadre très formalisé et très structuré. Toutefois, si l'on considère que l'ACA parvient à mettre en évidence un optimum objectif découvert par des voies dites scientifiques, alors l'identification de parties prenantes ou, tout simplement, la participation d'acteurs parties prenantes au processus n'a plus les mêmes raisons d'être. En effet, le calcul économique conduisant à la mise en évidence d'un optimum obtenu sur des bases objectives préexistantes à l'analyse, le résultat est indépendant (par définition) de la liste des acteurs participant au processus. Si l'on adopte ce point de vue, il y a lieu de s'interroger sur l'opportunité de faire participer différents acteurs car c'est, de surcroît, se heurter aux difficultés inhérentes à la participation d'acteurs multiples. Il s'ensuit qu'une concertation avec les parties prenantes n'a plus d'autres objectifs que celui de justifier une décision qui s'écarterait de cette approximation de l'optimum, laquelle doit servir de point de départ à ce débat public.

Toujours selon ce même point de vue, l'analyse coûts avantages pourrait tirer de l'objectivité et de la scientificité sensées fonder la capacité à rendre les décideurs publics plus responsables et plus attentifs aux attentes des parties prenantes. 
Même si l'on considère qu'il convient de "faire du bilan socio-économique, non le critère mais le noyau de l'estimation de la valeur d'un projet (...)» (CGP [1994]) il faut admettre que la valeur attribuée au projet par l'ACA ne peut pas se résumer à un simple chiffre mais doit être située dans un intervalle d'indétermination. Celui-ci a toutes chances d'être, dans bien des cas, assez grand. Dans ces conditions on est amené à s'interroger sur l'intérêt de l'estimation obtenue qui est, selon CGP [1994], « de permettre une analyse des raisons pour lesquelles on est conduit à s'écarter de la solution à laquelle ce seul bilan aurait conduit, et de pouvoir ainsi chiffrer le surcoût de la décision (...) ». Cette façon de raisonner ne paraît pouvoir engager la responsabilité des décideurs que si un projet unique émerge sur la base d'une estimation peu discutable de sa valeur, y compris par les autres parties prenantes.

Une éventuelle non conformation des décideurs à ce que prescrit l'ACA doit être interprétée, d'après ceux qui considèrent que cette expertise est apte à dégager un optimum, comme un écart par rapport à ce que dicte la rationalité la plus pure. Une telle déviation, qui ne peut qu'entraîner des surcoûts, nécessite donc d'être justifiée. En ce sens, l'analyse coûts avantages serait une démarche responsabilisante. Pour qu'une telle démarche fondée sur la mise en évidence d'un optimum servant de point d'ancrage puisse responsabiliser les diverses parties prenantes, il faut aussi qu'elles adhèrent suffisamment à la démarche. Cela implique qu'elles soient :

- aptes à en comprendre les grandes lignes ;

- disposées à admettre que, en dépit de ses imperfections, elle conduit effectivement à une bonne approximation d'un optimum objectif ;

- Convaincues de la pertinence des données qui conditionnent la détermination de cette approximation de l'optimum.

Ce sont là des conditions qui (si l'on tient compte des considérations développées au 4.1) paraissent difficiles à satisfaire lorsque l'on souhaite réunir des représentants de toutes les parties prenantes.

Selon nous, c'est avant tout une réelle compréhension de la démarche d'aide à la décision par l'ensemble des acteurs impliqués dans et par l'utilisation d'une telle démarche qui est le point déterminant de la légitimation de son résultat. La légitimité vient de ce qu'un acteur accorde ou non de la valeur et du crédit à quelque chose. Une démarche d'aide à la décision a du crédit lorsque les acteurs sont aptes à juger de la valeur de ce que permet de recommander ou de prescrire la démarche. Or, la complexité des méthodes de calcul, de pondération, d'actualisation et de monétarisation ne rendent compréhensibles les démarches d'analyse coûts avantages que par des techniciens et peu par les élus, et encore moins par d'autres parties prenantes telles que les associations d'usagers des transports ou des riverains concernés.

La longue liste des recommandations faites par le Syndicat des Transports d'Ile-de-France à usage des décideurs publics pour l'utilisation de l'ACA, de même que les recommandations de CGP [2001], mettent en évidence la complexité de ce mode d'analyse.

L'opacité des calculs de l'ACA renforce le caractère technocratique de la décision. Cela tient à deux raisons :

- l'instrumentation et le langage sont adaptés au mode de raisonnement et de prise en compte des systèmes de valeurs d'une catégorie particulière d'acteurs ; 
- cette instrumentation et ce langage sont trop souvent inintelligibles pour la majorité des autres acteurs.

Ainsi, le processus de décision publique en matière d'infrastructures de transport doit être conçu de façon à pouvoir faire face à des difficultés ayant une double provenance : d'un côté la difficulté d'instaurer un débat public structuré et utile à légitimer les décisions et, de l'autre, la difficulté d'instrumenter l'évaluation des projets d'investissements pour conduire au final à des choix qui soient d'une part pertinents du point de vue de l'utilisation rationnelle des ressources publiques et, d'autre part, acceptables du point de vue de la collectivité.

\section{A la recherche de nouveaux liens entre rationalité et légitimité}

La difficile institutionnalisation du débat public traduit en fait une absence historique d'expertise en matière de concertation. De ce fait, les démarches de concertation mises en œuvre ne conduisent pas souvent à une légitimation accrue des décisions. Parallèlement, l'évaluation des choix d'investissements est conduite en prenant appui sur un instrument très structuré et très formalisé. Celui-ci nécessite une expertise pointue, peu compatible avec des phases de débat successives. Cette double caractéristique de la décision publique met en évidence la présence de deux risques de dérive possibles du processus de choix des investissements de transport :

- une dérive technocratique caractérisée par la prépondérance d'outils d'évaluation mal adaptés au débat public et à la concertation ;

- la dérive de type 'foire d'empoigne' où le débat non structuré et non instrumenté ne permet d'atteindre que difficilement l'objectif initial et aboutit à la remise en cause partielle de la légitimité des décisions ou encore à la non décision, etc.

L'analyse qui précède conduit à s'interroger sur les modalités à concevoir pour aménager processus et outils dans le but d'échapper à ces deux risques possibles et pour répondre conjointement et de façon cohérente aux deux catégories d'enjeux présentées en introduction : rationaliser l'utilisation des ressources publiques et parvenir à des choix acceptables du point de vue de la collectivité, autrement dit comment réconcilier rationalité et légitimité.

Pour y parvenir, il est indispensable de prendre en compte le fait que les concepteurs ont le plus souvent une connaissance pointue des projets qu'ils ont contribué à élaborer. Il en va de même pour les évaluateurs. Ceci crée une asymétrie entre les techniciens et les autres parties prenantes qui ne sont pas impliquées de la même façon dans ces phases de conception et d'évaluation. Cette asymétrie peut entraver le débat et même le bloquer. L'aménagement de la phase amont telle qu'elle a été proposée dans DTT [2004] est sans doute un moyen d'atténuer les effets négatifs de cette asymétrie mais cela implique que certaines conditions soient respectées :

- pouvoir tirer profit, pour l'évaluation socio économique, des éléments du débat public de phase amont et disposer d'outils structurant le débat ;

- décloisonner la phase de calcul économique survenant en aval et celle de débat public se produisant en amont.

Pour achever cet article, nous prenons le risque de faire diverses propositions (dont certaines pourront paraître utopiques). Elles visent à mieux concilier la poursuite des deux objectifs rappelés au début de la présente section. Elles se situent dans une double perspective. Elles 
concernent en premier lieu l'aménagement possible du processus de choix des investissements et l'intégration d'outils d'évaluation nouveaux et adaptés au type de processus proposé. Elles concernent en second lieu l'ACA proprement dite dont nous discutons la place et le rôle à la fois en amont et en aval du processus concerné.

\subsection{Aménagement du processus et renouvellement des outils}

L'évaluation socio-économique est, nous l'avons évoqué plus haut, mal adaptée au débat public. Elle a en revanche l'avantage de constituer un cadre formel bien structuré qui peut servir de référent pour uniformiser les pratiques. Le premier groupe de travail Boiteux avait d'ailleurs pour objectif principal de proposer un cadre référent pour des pratiques qui, à l'époque, étaient très peu homogènes d'une administration à une autre. Le recours au calcul économique est néanmoins source de dysfonctionnements importants, essentiellement la mise en position asymétrique des experts des méthodes d'ACA et des autres parties prenantes, l'occultation de ce fait d'une partie des bienfaits du débat public et, finalement, la réduction du choix entre projets à la seule question de leur évaluation socio-économique. Dans ce contexte, le processus laisse peu de place au débat public et rend difficile la mise en œuvre d'une concertation effective. Or, il nous semble que la mise en œuvre d'une telle concertation soit au cœur de la problématique de l'évolution des contextes décisionnels évoquée plus haut. Comme nous allons l'expliquer, celle-ci devrait prendre la forme d'une table de concertation apte à permettre suffisamment tôt l'expression et le recueil des points de vue sur les objectifs et grandes lignes des projets en cours d'élaboration. Dans cette phase amont, la concertation doit bien évidemment rester compatible avec les exigences de l'évaluation économique mais elle doit mobiliser d'autres types d'outils d'évaluation que l'ACA.

\subsubsection{Mise en place d'une table de concertation}

La brève référence à l'évolution historique de l'institutionnalisation du débat public a montré (cf. 3.1.) que le législateur a tenté de répondre aux exigences des nouveaux contextes décisionnels. DTT [2004] est elle même en partie consacrée à la recherche d'une «meilleure manière d'organiser la complémentarité entre évaluation et concertation ». Cet objectif conduit à s'interroger sur les modalités de mise en place de ce que nous proposons d'appeler une table de concertation. Ce terme, déjà en usage au Québec, est utilisé ici pour souligner que ce qu'il s'agit d'organiser est autre chose que le débat public tel qu'il est aujourd'hui pratiqué en France. La table de concertation doit être, selon nous, l'instrument d'une démarche visant à atteindre les objectifs suivants (cf. Damart [2003]) :

- provoquer et organiser la participation de l'ensemble ou d'une grande partie des acteurs détenteurs d'enjeux par rapport au problème de décision posé ;

- mettre en œuvre un processus permettant l'expression de tous et une réelle prise en compte des points de vue de tous les acteurs impliqués ;

- mettre en évidence les systèmes de valeurs et d'objectifs que chacune des parties prenantes tentent de faire valoir ;

- faire émerger, malgré les divergences de vues et d'objectifs, des éléments de consensus et, au final, des choix qui recueillent l'assentiment de tous les acteurs.

Si l'on accepte que ces objectifs définissent ce que l'on entend par concertation, alors la mise en œuvre de processus qui associeraient habilement évaluation et concertation conduit à plusieurs préoccupations. 
En premier lieu, la mise en place d'une table de concertation mérite d'être considérée comme une activité aux enjeux spécifiques. Elle devrait être placée sous la conduite d'un maître d'œuvre, facilitateur comme l'on dit au Québec, dont le rôle consiste à assurer que soient atteints les objectifs d'une table de concertation cités plus haut. La question se pose alors de savoir comment est nommé un tel facilitateur autrement dit par qui. La nature du rôle de cet acteur contraint, pour des impératifs évidents de neutralité, à une séparation entière entre d'une part le responsable de l'organisation de la table de concertation et, d'autre part, le maître d'ouvrage du ou des projet d'investissement soumis à évaluation. Le premier ne saurait être le même acteur que le second et, surtout, le premier ne saurait être nommé par le second. Il semblerait a priori raisonnable que la CNDP, compte tenu de ses fonctions, puisse jouer ce rôle de maître d'œuvre de la table de concertation. Néanmoins, les points faibles de cette commission mentionnés au 3.2.2. et les conditions fortement contraintes dans lesquelles celleci est aujourd'hui saisie ne nous semblent pas de nature à justifier qu'un tel rôle puisse lui être systématiquement attribué.

En second lieu, la participation à la table de concertation d'acteurs multiples nécessite une phase préalable de réflexion sur la liste des acteurs à impliquer ainsi que sur la façon dont ils peuvent ou doivent être représentés. Cette phase est importante car la participation des acteurs parties prenantes est un des fondements de la concertation et une mauvaise ou incomplète identification ou représentation des parties prenantes peut amener à discréditer une partie du processus. Par ailleurs, nous nous posons la question de savoir qui doit décider de l'étendue de la participation et des acteurs à impliquer. Il paraîtrait raisonnable que cette tâche fasse partie des attributions du responsable de l'organisation de la table de concertation. Enfin, l'étendue de la participation en phase amont du processus doit-elle rester invariante tout au long de celui-ci ? Ne faut-il pas envisager que la composition de la table de concertation puisse évoluer?

En dernier lieu, faut-il envisager la table de concertation comme une démarche uniquement dédiée à la phase amont du processus plus global de choix des investissements publics ? En cohérence avec le souci de coupler plus étroitement le débat public et l'évaluation, il nous paraît opportun de faire de la concertation et de l'évaluation deux démarches glissantes dont l'importance et la nature varient au cours du processus. En phase amont, ce serait la concertation qui prendrait le pas, attachée à une démarche d'ACA adaptée (cf. 5.2.1.) et à d'autres outils d'évaluation plus facilement mobilisables que l'ACA dans cette partie du processus. En amont, il parait pertinent de faire porter le débat sur des points qu'une ACA ne permet pas d'éclaircir : par exemple les réponses à la question " pourquoi ce projet ? », les caractéristiques générales des projets en cours d'élaboration, les objectifs généraux, les contraintes fortes, les variantes envisageables, etc. Dans une partie aval du processus, la concertation changerait de nature et d'importance, laissant plus de place à l'évaluation socio-économique proprement dite mais tout en conservant la possibilité d'influencer ou même de remettre en cause les résultats de l'évaluation en prenant notamment en compte des aspects qualitatifs (impacts sur l'environnement ou l'aménité des paysages par exemple).

\subsubsection{Instrumentation des premières étapes de la concertation}

Avant l'évaluation des projets proprement dite, il est nécessaire de fournir, lors de la phase amont du processus, des outils spécifiquement dédiés à la structuration des échanges et de l'expression des points de vue de tous les acteurs impliqués. La lente institutionnalisation du débat public a entraîné un retard important en termes d'instrumentation du débat. Une concertation implique la participation d'acteurs multiples et suppose que les points de vue de tous 
soient, sous une forme ou une autre, pris en compte. Il existe des outils pour organiser cette concertation en facilitant les interactions entre acteurs. Les cartes cognitives sont un exemple de ce type d'outils. Elles ont notamment été utilisées avec succès dans le cadre d'un processus de décision public portant sur une question d'aménagement du territoire et de développement durable (cf. Amorim [2000]). Les cartes cognitives sont des représentations graphiques très simplifiées construites, sur la base du discours des acteurs, sur leur façon de concevoir un problème de décision. Concernant le problème de choix de projets d'infrastructures de transport, cette représentation peut être celle des différents effets perçus du projet et des liens de cause à effet correspondants. La visualisation des préférences et des points de vue des différents acteurs parties prenantes facilite ensuite la formalisation du problème et peut servir de base à un débat structuré. L'application des cartes cognitives qui vient d'être évoquée concernait un ensemble de communes qui devaient s'accorder sur l'élaboration et la mise en place d'un programme de développement durable intercommunal (programme portant le nom « d'Agenda 21 »). Une première carte cognitive a permis de représenter visuellement la structure du processus de concertation envisagée par chaque acteur. La carte a ainsi matérialisé la perception de chacun sur les finalités, les outils, les étapes, les analyses et les recensements réalisés dans le cadre de l'état des lieux et du diagnostic, les étapes de concertation et de décision politiques au niveau intercommunal, les politiques, projets et modalités retenus après expertise, les différents publics concernés, des flèches indiquant des liens de cause à effet ou des liens temporels. Cette carte cognitive avait ainsi pour objectif de dégager une vision globale du processus à partir de laquelle chacun pouvait se situer et agir. Une seconde carte cognitive a été construite, cette fois pour représenter la perception pour chacun des enjeux liés au développement durable proprement dit et non le processus de concertation. Il s'agissait là de recueillir le plus d'informations possible sur les besoins et attentes de chacun, les opportunités et les contraintes en matière de développement durable sur la région concernée.

Il existe d'autres outils pour organiser la concertation et faciliter les interactions entre acteurs (cf. Damart [2003]). Nous n'en évoquerons ici qu'un autre, les Group Decision Support Systems (GDSS). Un GDSS est un système composé d'un animateur ou facilitateur, d'une méthodologie et de un ou plusieurs ordinateurs équipés de logiciels ad hoc. Un GDSS est essentiellement dédié à la facilitation de la décision en groupe. Les développements récents des nouvelles technologies de l'information et de la communication ont sensiblement contribué à étendre les recherches sur ce type d'outils et à diffuser leur utilisation dans un certain nombre de contextes de décision. Aujourd'hui, les nouvelles technologies laissent entrevoir de multiples possibilités en termes de structuration et d'organisation des débats. En 1991, le problème du bouclage de l'autoroute dite francilienne a même donné lieu à la réalisation d'une plateforme multimédia de formation à la pratique de la concertation dans le cadre de problèmes de décision portant sur des infrastructures de transport (cf. De Carlo et Choulet [2003]).

La structuration des échanges permet, grâce à ce type d'outils, de formaliser des points de vue. Ce travail a l'avantage de donner du crédit à la démarche de concertation et a également l'avantage de servir de support pour la construction de critères sur la base desquels une évaluation des projets en phase amont est possible.

\subsubsection{Renouvellement des outils d'évaluation en phase amont}

Les méthodologies de l'analyse coûts avantages constituent (ce qui est un point fort) un cadre de référence homogène pour l'évaluation des projets d'investissements. En contre-partie, telle qu'elle est pratiquée aujourd'hui, l'ACA présente le défaut d'une technicité importante et d'un formalisme qui la rend difficilement intégrable au sein d'un débat public. Par ailleurs, la 
volonté du législateur de prendre explicitement en compte dans l'évaluation des projets des éléments par nature difficilement quantifiables et monétarisables laisse la place à d'autres outils d'évaluation que l'ACA. Ces outils, complémentaires à l'ACA, répondraient idéalement à deux catégories d'enjeux : servir de support au débat public et finalement à la concertation d'une part et permettre que soient pris en compte les effets d'un projet que l'ACA ne peut pas intégrer d'autre part.

La méthodologie multicritère apporte de tels outils. Elle repose sur une démarche d'évaluation significativement différente de celle mise en jeu dans l'ACA. Dans cette dernière en effet, il est indispensable (cf. section 2.2.) :

$1 /$ de quantifier tout ce qu'il s'agit de prendre en compte ;

2/ d'attribuer une valeur monétaire aux unités de chacune de ces quantités.

Aucune de ces deux exigences ne s'impose dans une démarche d'évaluation multicritère. Celle-ci doit être conduite de façon à prendre en compte l'ensemble des points de vue jugés pertinents au travers de ce qui est appelé une famille cohérente de critères (cf. Roy [2000]).

Chacun des critères d'une telle famille doit être conçu pour permettre une évaluation des projets soit dans une unité concrète lorsqu'elle peut être clairement intelligible par les acteurs (nombre de morts, d'heures, de tonnes équivalent $\mathrm{CO} 2$, etc.), soit sur une échelle qualitative appropriée aux points de vue pris en compte ${ }^{4}$. L'évaluation sur plusieurs dimensions ainsi obtenue ne masque pas prématurément l'hétérogénéité des conséquences qu'il s'agit de prendre en compte pour éclairer la décision. De surcroît, l'imprécision, la mauvaise détermination, la part d'arbitraire qui peuvent affecter l'évaluation sur chaque critère peuvent aisément être explicitées par des seuils d'indifférence et de préférence (cf. Roy [2000]). Au début de la phase amont, le tableau des évaluations ainsi bâti peut n'être que partiellement rempli et, malgré cela, utile à la table de concertation.

Divers types d'outils ont en outre été conçus pour exploiter ce tableau dans une perspective de faciliter les comparaisons entre les projets ainsi évalués. Ces outils relèvent essentiellement de deux types d'approches. Le premier consiste à construire, sur la base de l'évaluation multicritère, un critère unique de synthèse. Celui-ci peut être la valeur actuelle nette ou le taux de rentabilité interne comme l'a fait l'ACA (cf. section 2.2.2.). Toutefois, l'ACA n'explicite ni la famille cohérente de critères, ni le tableau des évaluations qui lui est attaché (avec leurs unités propres), pas plus que les seuils d'indifférence et de préférence.

Le second type d'approche conduit à simplement faire la comparaison paire par paire des projets envisagés sur différents critères. Certaines méthodes relevant de ce second type considèrent ainsi les critères comme des "votants » exprimant des points de vue sur chaque comparaison. Dans ce type d'approche, il n'est nul besoin de parvenir à quantifier les évaluations sur les critères. Il n'est nul besoin également d'exprimer toutes les évaluations en une même unité (monétaire par exemple).

Dans le cadre des démarches de concertation, les outils multicritères pourraient, à notre avis, contribuer à mieux légitimer les décisions. Pour justifier ce point de vue, nous voudrions attirer l'attention sur les points suivants :

\footnotetext{
${ }^{4}$ Dans ce dernier cas, les difficultés que l'on peut rencontrer pour définir ce que recouvrent les échelons d'une telle échelle constituent un bon exercice permettant de bien clarifier ce qui est en question.
} 
- Il s'agit d'outils permettant de prendre en compte explicitement, sans recours à un formalisme excessif, plusieurs critères parfois de nature très différente. La comparaison paire par paire de projets peut être réalisée sur la base de l'évaluation de ces projets sur des critères quantitatifs et qualitatifs. Il est même possible de s'affranchir d'un recodage numérique pour les critères qualitatifs.

- La quantité d'information préférentielle et de paramètres qui permettent de faire fonctionner de tels outils est peu importante, si bien que la part d'arbitraire nécessairement contenue dans tout outil d'aide à la décision est dans ce cas limitée.

- L'utilisation d'outils multicritères n'est pas incompatible avec l'évaluation socio-économique. La possibilité de prendre en compte des critères qualitatifs que l'on ne cherche pas à quantifier et à monétariser (critères appréciant l'impact d'un projet sur l'environnement ou sur l'esthétique d'un paysage par exemple) ne doit pas être interprétée comme une façon d'occulter des aspects financiers ou économiques.

- La pratique de l'aide multicritère à la décision peut être aisément appropriée par de multiples acteurs, y compris ceux qui n'en sont pas experts. De ce point de vue, elle constitue ainsi un outil sur lequel s'appuyer pour structurer une concertation et un débat autour des enjeux posés par différents projets (cf. notamment Froger et Oberti [2002]).

- Dans certaines méthodes d'aide multicritère à la décision, l'agrégation des différents points de vue pour comparer deux projets fait intervenir non seulement la position de ceux qui expriment une opinion majoritaire (compte tenu d'une pondération des points de vue) mais aussi celle de ceux qui sont minoritaires ${ }^{5}$ lorsqu'ils sont en forte opposition (possibilité de veto). On peut voir là un principe de démocratie raisonnée apte à contribuer à mieux légitimer les décisions.

\subsection{Renouvellement des modes d'utilisation des outils d'expertise actuels}

Les outils multicritères dont il vient d'être question pourraient être utilisés conjointement avec ceux du calcul économique. Toutefois, la façon de concevoir cette complémentarité dépend des objectifs visés, laquelle évolue au cours du processus de concertation. Nous faisons ciaprès quelques propositions dans ce sens en reprenant la distinction phase amont (cf. 5.2.1) et phase aval (cf. 5.2.2) bien que, comme cela a déjà été mentionné, il n'y a pas de séparation très nette entre ces deux phases.

\subsubsection{Pratique de l'ACA en phase amont}

Les défauts des outils d'ACA dans le cadre d'une concertation ne doivent pas conduire à rejeter l'ACA qui fournit par ailleurs, pour l'évaluation des projets, un support formel qui sert de référent à toute administration impliquée dans un processus de décision publique. Les évolutions des contextes décisionnels doivent néanmoins conduire à une réflexion sur la façon dont

\footnotetext{
${ }^{5}$ Dans ces méthodes, la prise en compte des positions minoritaires n'a pas le caractère d'une simple compensation d'inconvénients par des avantages d'une autre nature comme c'est le cas avec l'ACA (cf. Roy et Damart [2002]).
} 
est pratiquée 1'ACA et sur les modalités du couplage débat - évaluation ${ }^{6}$. Le législateur a récemment milité pour un resserrement des liens entre débat public et évaluation socioéconomique des projets. Il n'en précise cependant pas les modalités et reste vague sur les contributions du débat aux phases d'évaluation socio économique des projets.

Il nous semble opportun de considérer le débat et l'évaluation - non restreinte à l'ACA comme faisant parti d'un tout indissociable. Ainsi le débat n'est pas réduit à une formalité qui permet de s'assurer de l'acceptabilité sociale de projets qui ont déjà été évalués. De même, l'information que fournit une évaluation formelle des projets n'est pas sans incidence sur un débat et une concertation qui se fixerait comme objectif de prendre explicitement en compte tous les points de vue, y compris les points de vue économiques. Nous suggérons ainsi que l'évaluation socio-économique soit envisagée comme une démarche glissante le long d'un processus qui intègre d'autres outils plus adaptés au débat que ne l'est l'ACA pour les raisons évoquées plus haut.

En phase amont, l'ACA pourrait ainsi être utilisée dans une version allégée qui ne mette pas en situation d'asymétrie les experts de l'analyse économique et les autres parties prenantes. Lors de cette phase amont, il pourrait être discuté des grandes catégories d'effets à prendre en compte et des outils multicritères pourraient ici jouer un rôle majeur en permettant de formaliser les différents points de vue et, surtout, de réaliser une première évaluation des projets. La contribution de cette partie du processus résiderait ainsi dans la possibilité de faire participer directement à l'activité d'évaluations des projets en concurrence des parties prenantes qui n'ont traditionnellement aucune possibilité de faire valoir un point de vue dans le cadre de cette activité. Autrement dit, une telle organisation de la phase amont permettrait de réconcilier les exigences de rationalité de l'expertise avec celles de légitimité du débat public. Le fait qu'il y ait débat sur la façon dont les évaluations sont conduites et formalisées dans le cadre d'une famille cohérente de critères nous paraît être de nature à mieux faire comprendre et à accepter la façon dont les résultats ainsi obtenus sont intégrés dans le calcul de l'ACA et, corrélativement, à relativiser le rôle qu'il convient de faire jouer aux évaluations qui ne peuvent y être intégrées.

En phase aval, les techniques d'ACA retrouvent toute leur pertinence à condition que leur pratique puisse être enrichie. C'est ce dont il va être question.

\subsubsection{Enrichir les techniques d'ACA en phase aval}

L'information fournie par une ACA est riche tant le nombre d'effets induits par un projet et pris en compte dans l'évaluation est important. Pour autant, cette richesse a pour corollaire la nécessité de définir un grand nombre de paramètres (cf. 2.2.1.). Nous avons vu que l'attribution de valeurs numériques aux paramètres n'était pas exempte d'arbitraire. Cette présence ne remet pas en cause l'utilité de l'ACA. Cependant, telle que l'ACA est pratiquée, la notion d'arbitraire est niée ou, au mieux, ramenée à de l'imprécision non réellement prise en compte. En particulier, les tests de sensibilité proposés par le législateur ou recommandés par le CGP ne sont pas de nature à prendre raisonnablement en compte l'arbitraire contenu dans les démarches d'ACA. En effet, ils consistent, tels qu'ils sont pratiqués aujourd'hui, à faire varier à la marge et un par un différents paramètres du bilan socio-économique : le taux d'actualisation lorsque le terme du projet est long par exemple. Cet exercice permet de voir ainsi si le taux de rentabilité d'un projet bascule et passe sous la barre d'un taux acceptable

\footnotetext{
${ }^{6}$ Réflexion encouragée par DTT [2004].
} 
lorsque l'on fait varier un paramètre. La prise en compte de l'arbitraire contenu dans le paramétrage du bilan pourrait être améliorée si l'on procédait plutôt à des tests de robustesse. Ceux-ci consisteraient à faire varier simultanément différents paramètres du bilan socio économique. Prenons l'exemple de deux projets A et B. Un test de robustesse pourrait par exemple consister à prendre les conditions de paramètres et de situation de référence les pires pour le projet $\mathrm{A}$ et les pires pour le projet $\mathrm{B}$ et à observer si les préférences en termes de valeur actuelle nette s'inversent ou non par rapport à celles découlant de valeurs centrales ou de valeurs les plus favorables.

Une autre façon d'enrichir la pratique de l'ACA serait, dans une phase aval, d'un processus similaire à celui suggéré précédemment, de faire apparaître explicitement les effets les plus déterminants dans l'évaluation d'un projet (tels que, comme c'est le cas bien souvent, le nombre d'heures réputées gagnées), puis de les démonétariser de façon rendre de nouveau accessible l'évaluation du projet aux différentes parties prenantes. Dans le même esprit, il pourrait être intéressant de mettre également en évidence l'impact négligeable (compte tenu de la façon dont ils sont pris en compte) que peuvent avoir certains facteurs tels que la pollution, le bruit, les effets de coupure, la sécurité,... La monétarisation masque en effet les compensations discutables qui s'opèrent entre des inconvénients de cette nature et des avantages par ailleurs contestables tels que les gains de temps. Ainsi, tests de robustesse, démonétarisation nous paraissent être de nature à consolider encore un peu plus le couplage débat-évaluation.

\section{Conclusion}

L'évolution des contextes décisionnels liés aux infrastructures de transport est telle que, désormais, le décideur public est constamment soumis aux exigences de deux catégories de préoccupations : rationaliser l'utilisation des ressources publiques et faire des choix acceptables du point de vue de la collectivité des acteurs parties prenantes.

L'évaluation socio-économique des projets fondée sur les techniques d'analyse coûts avantages est pratiquée dans le but de satisfaire à la première catégorie d'enjeux. Nous en avons décrit les principes et nous avons tenté de montrer comment elle était pratiquée.

La seconde catégorie a conduit le législateur à progressivement institutionnaliser des phases et des outils dédiés au débat public. Nous avons en particulier présenté les fonctions de l'enquête publique préalable et de la Commission Nationale du Débat Public.

Au cours de cet article, nous avons été amenés à défendre la thèse selon laquelle l'analyse coûts avantages, telle qu'elle est actuellement pratiquée, permet difficilement de servir un débat public pertinent et constructif. En fait, tenter de satisfaire les deux préoccupations rappelées ci-dessus, c'est tenter de structurer une articulation plus étroite entre évaluation et débat. Ce couplage peut aujourd'hui être amélioré comme nous avons tenté de le montrer en dernier lieu dans cet article. Derrière cette question, ce sont plus généralement les rapports entre les exigences de rationalité que les acteurs aimeraient voir attachées à tout processus de décision d'une part et les exigences de légitimité de fait liées à tout processus collectif d'autre part qui sont questionnés. Ces exigences ne sont pas incompatibles. Selon nous, le possible renouvellement de l'instrumentation de l'évaluation socio-économique ou de la façon de pratiquer le calcul économique que nous avons exposé le montre. 


\section{Références}

Agard J. (ed.), 1970, Rationalisation des choix budgétaires, Paris, Dunod, Monographies de recherche opérationnelle $\mathrm{n}^{\circ} 12$.

Amorim N., 2000, Aide à la concertation et à la décision dans le cadre de processus de décision publique complexes, Thèse de doctorat, Université Paris-Dauphine.

Andan O., Faivre-D’arcier B., Hiron B., 1995, Mesure du consentement à payer pour l'environnement : analyse sur les méthodes d'enquête. Rapport pour l'INRETS, Commande LEN n 20495040, juin 1995.

Auzannet P., 1997, Quelle méthode d'évaluation pour les transports en milieu urbain ?, Transport public, janvier 1997.

Barraque B., 2002, Quels indicateurs de gêne et quelle démarche participative pour quelle politique territoriale d'aéroports ?, Responsabilité et Environnement, $\mathrm{n}^{\circ} 26$, avril 2002.

Blanc H., 1998, La commission nationale du débat public : un nouvel âge de la concertation en amont ?, Les Annales des Mines « Responsabilité et Environnement » $\mathrm{n}^{\circ} 9$.

Cahen B., Colombo F., 1999, Les décisions de transport en Ile de France : rationalité perdue et désordre démocratique, Mémoire d'Ingénieurs du Corps des Mines sous la direction de François Engel (Ecole des Mines de Paris).

CGP, 1994, Transports : pour un meilleur choix des investissements, Commissariat Général au Plan, La Documentation Française.

CGP, 2001, Transports : choix des investissements et coût des nuisances, Commissariat Général au Plan, La Documentation Française.

Damart S., 2003, Une étude de la contribution des outils d'aide à la décision aux démarches de concertation. Le cas des décisions publiques de transport, Thèse de doctorat, Université Paris-Dauphine.

De Carlo L., Choulet J.P., 2003, Quelle place pour le multimédia dans la formation à la concertation? L'exemple du Cd Rom La Francilienne, Concertation, Décision et Environnement, vol II, Paris, La Documentation Française.

DTT, 2004, Instruction cadre du 25 mars 2004, Direction des Transports Terrestres.

Falque M., 1987, Analyse critique et prospective des études d'impact, in Cahier du Germes $\mathrm{n}^{\circ}$ 12, L'environnement dans l'analyse et la négociation des projets, pp. 521-538.

Fourniau J.-M., 2002, Concurrence entre collectivités ou débat public : les ambiguïtés de la DUCSAI, Responsabilité et Environnement, $\mathrm{n}^{\circ}$ 26, avril 2002.

Froger G., Oberti P., 2002, Gouvernance et développement durable: l'aide multicritère à la décision participative, Revue Sciences de la Société, Autour du développement durable, $\mathrm{n}^{\circ} 57$, octobre 2002, pp. 57-74 
Hammiche S., Denant Boemont L., mars 1997, Cohérence du calcul économique et financement public des grands projets d'infrastructure : le cas de l'autoroute ferroviaire, La Revue Economique, vol. 48 n 2, pp. 271-294.

Lascoumes P., 2002, La DUCSAI, quel exemple de débat public ?, Responsabilité et Environnement, $\mathrm{n}^{\circ} 26$, avril 2002.

Le Duff R., Papillon J.C., 1989, Gestion du non-marchand, Encyclopédie de Gestion, sous la direction de Patrick Joffre et Yves Simon, Economica, 1424-1447.

Molines N., 2003, Méthodes et outils pour la planification des grandes infrastructures linéaires et leur évaluation environnementale, Thèse de doctorat, Université Jean Monnet de SaintEtienne.

Plottu E., 1998, Evaluation et prise de décision : impacts socio-économiques et environnementaux dans les grands projets d'infrastructure de transport, Revue d'Economie Régionale et Urbaine, $\mathrm{n}^{\circ} 2$, pp. 301-320.

Roy B., 2000, L'aide à la décision aujourd'hui : que devrait-on en attendre ? in Les nouvelles fondations des Sciences de gestion - Eléments d'épistémologie de la recherche en management, ouvrage coordonné par Albert David, Armand Hatchuel et Romain Laufer, Librairie Vuibert, Collection FNEGE, pp. 141-174.

Roy B., Damart S., 2002, L'analyse coûts avantages, outil de concertation et de légitimation ?, Metropolis, $\mathrm{n}^{\circ} 108 / 109$, pp. 7-16. 